\title{
Experimental study of large-amplitude perturbations in space-charge dominated beams
}

\author{
K. Tian, ${ }^{*}$ R. A. Kishek, I. Haber, M. Reiser, and P. G. O'Shea \\ Institute for Research in Electronics and Applied Physics, University of Maryland, College Park, Maryland 20742, USA
}

(Received 12 May 2009; published 8 March 2010)

\begin{abstract}
Detailed experimental measurements are presented concerning the propagation of space-charge waves of varying amplitudes in an intense, charged-particle beam. A short perturbation to the density profile is applied at the electron gun, and both current and mean energy profiles are measured at two locations downstream. The measurements are compared to predictions of a linear 1D cold-fluid model, and selfconsistent particle-in-cell simulations. For sufficiently small perturbation amplitudes, the experiment, simulation, and 1D theory agree. For larger amplitudes, the simulation begins to diverge from theoretical predictions due to nonlinear effects. Experimental observations for large-amplitude perturbations differ markedly from either theory or simulation. With the aid of simulations with mismatched and misaligned beams, this departure of experiments from predictions is demonstrated to be caused by the loss of beam current due to scraping aided by the larger radius of the perturbation.
\end{abstract}

DOI: 10.1103/PhysRevSTAB.13.034201

PACS numbers: 29.27.Bd, 29.27.Fh, 29.30.Dn

\section{INTRODUCTION}

Many modern applications of intense particle beams require the transport of high intensity beams over substantial distances without significant degradation of beam quality. For most of these machines, especially near the source and injector, the internal repulsion due to space-charge forces can introduce nonlinear forces that cause a reduction in beam quality. One example is the longitudinal spacecharge waves that are generated by a perturbation to the beam density or energy resulting from small errors in the applied fields. Such perturbations can lead to instabilities that disrupt the beam under certain circumstances. For example, in electron machines, coherent synchrotron radiation can be generated from a density-modulated beam in a bend, causing the growth of energy spread and emittance [1-3], beam instability, and microbunching [4-6]. Hence, in order to preserve high beam quality, it is important to understand the longitudinal space-charge beam dynamics in the injector region. For proton and ion accelerators, space-charge effects persist for a far longer distance from the source, making the study of space-charge waves important for manipulating or compressing the bunch longitudinally, and for controlling instabilities. As a result of growing interest in intense beam applications such as accelerator-driven high-energy-density physics [7], pulsed neutron sources [8], and x-ray free electron lasers [9], a detailed knowledge and understanding of space-charge waves has become increasingly important for the successful operation of such machines.

There have been recent efforts in studying space-charge dominated beams experimentally; for example, the high

\footnotetext{
*kaitian@umd.edu

Present address: Thomas Jefferson National Accelerator Facility, Newport News, VA 23606.
}

current experiment (HCX) $[10,11]$ and the Paul trap simulator experiment (PTSX) [12]. Since the early 1990s, the charged particle beams group at University of Maryland has carried out numerous experimental studies on longitudinal space-charge waves by systematically introducing localized density or energy modulations into highly intense electron beams [13-15]. When the initial modulations are relatively small compared with unperturbed values, a linear 1D cold-fluid model has been found to be adequate to reproduce the experimental results [13]. However, the accuracy of these studies is limited in some aspects. For example, precise measurements of the mean energy profiles were unavailable prior to the introduction of a highresolution energy analyzer $[16,17]$. For larger-amplitude perturbations, the linear cold-fluid model has been found not be adequate in predicting beam behavior [18,19]. Furthermore, this 1D model neglects the consequences of the transverse distribution on the longitudinal dynamics.

Aiming to resolve these issues by means of more detailed measurements and self-consistent particle-in-cell (PIC) simulations, we have built a dedicated experimental system, the long solenoid experiment (LSE), to probe the physics of space-charge dominated beams, particularly longitudinal space-charge waves. Since the initial conditions of the beam are critical for the accurate prediction of the beam behavior, it is important to measure the initial beam conditions for both the perturbed and unperturbed beams. The typical width of the perturbation is only about $10 \mathrm{~ns}$. Therefore, the measurements must resolve that time scale. Previously, we have reported the measurement of both the detailed mean energy profiles [20], and timeresolved transverse distributions [21] of space-charge waves. These results have informed on the expected correlations between the transverse and longitudinal beam distributions. More importantly, these measurements can be used to initialize self-consistent simulations. In this paper, 
we present new findings on large-amplitude perturbations from experimental measurement and numerical simulations.

In Sec. II, we briefly review the 1D cold-fluid theory used for modeling the propagation of the linear spacecharge wave. In Sec. III, we describe the LSE system and the experimental method of generating controlled perturbations. We then present new experimental measurements of space-charge waves originating from large-amplitude perturbations. This is followed in Sec. IV by a systematic simulation study of using the particle-in-cell code WARP [22]. Finally, Sec. V concludes with a summary and a description of remaining issues.

\section{1D COLD-FLUID MODEL FOR SPACE-CHARGE WAVES}

To fully understand and model the longitudinal dynamics of space-charge waves, one should carry out 3D analysis that includes the effects from transverse dynamics and finite beam temperature. Nevertheless, a 1D cold-fluid model is sufficiently accurate for beams satisfying the long wavelength limit, where the wavelength of the perturbation is much larger than the transverse beam radius.

In this model, the beam is considered to be an infinitely long cylinder with line charge density of $\Lambda_{0}$ and radius $a$ inside a conducting drift tube of radius $b$. We assume that the perturbation is much smaller in density and velocity than the unperturbed beam and neglect the longitudinal momentum spread of the beam. Thus, the continuity and momentum fluid equations are used to describe the beam dynamics, with an assumption of zero temperature used to truncate the hierarchy. The 1D cold-fluid model shows that a small density or/and velocity perturbation stimulates two space-charge waves, the fast wave and the slow wave, moving in opposite directions in the beam frame with the same phase velocity, $c_{s}$, which is also called "sound speed" and defined as

$$
c_{s}=\sqrt{\frac{q g \Lambda_{0}}{4 \pi \varepsilon_{0} \gamma_{0}^{5} m}}
$$

where $q$ is the particle charge, $m$ is the particle mass, $\varepsilon_{0}$ is the vacuum permittivity, $\gamma_{0}$ is the Lorentz factor, and $g$ is a geometry factor, characterizing the relationship between the longitudinal self-electric field and the variation of the line charge density, and has been discussed thoroughly in Refs. [14,23]. For infinitely long space-charge dominated beams, the $g$ factor can be expressed as $g=2 \ln (b / \bar{a})$, where $\bar{a}$ is the average beam size. Since the phase velocity is independent of frequency, both fast and slow waves are nondispersive and preserve their shapes during propagation.

The 1D cold-fluid model has been solved in the lab frame by keeping only the first order terms [13]. The solutions of the perturbation of line charge density $\Lambda_{1}$, velocity $v_{1}$, and current $I_{1}$ are expressed as

$$
\begin{aligned}
& \Lambda_{1}(z, t)=-\frac{\Lambda_{0}}{2}\left[\delta \frac{v_{0}}{c_{s}}-(\eta-\delta)\right] f\left(t-\frac{z}{v_{0}-c_{s}}\right)+\frac{\Lambda_{0}}{2}\left[\delta \frac{v_{0}}{c_{s}}+(\eta-\delta)\right] f\left(t-\frac{z}{v_{0}+c_{s}}\right), \\
& v_{1}(z, t)=\frac{v_{0}}{2}\left[\delta-(\eta-\delta) \frac{c_{s}}{v_{0}}\right] f\left(t-\frac{z}{v_{0}-c_{s}}\right)+\frac{v_{0}}{2}\left[\delta+(\eta-\delta) \frac{c_{s}}{v_{0}}\right] f\left(t-\frac{z}{v_{0}+c_{s}}\right), \\
& I_{1}(z, t)=-\frac{I_{0}}{2}\left[\delta \frac{v_{0}}{c_{s}}-\eta+(\eta-\delta) \frac{c_{s}}{v_{0}}\right] f\left(t-\frac{z}{v_{0}-c_{s}}\right)+\frac{I_{0}}{2}\left[\delta \frac{v_{0}}{c_{s}}+\eta+(\eta-\delta) \frac{c_{s}}{v_{0}}\right] f\left(t-\frac{z}{v_{0}+c_{s}}\right),
\end{aligned}
$$

where subscripts 0 and 1 represent the unperturbed and perturbed physical quantities, respectively. The initial localized velocity perturbation and current perturbation are in the form

$$
v_{1}(0, t)=\delta v_{0} f(t) \quad I_{1}(0, t)=\eta I_{0} f(t),
$$

where $\delta$ is a small, positive quantity to specify the strength of the velocity perturbation; $\eta$ is a small quantity to specify the strength of the initial current perturbation; $f(t)$ is any smooth function with an amplitude of unity which represents the shape of the perturbation and is supposed to vanish when $t$ is equal or smaller than zero. In each of the equations of (2), the first term is the slow wave; the second term is the fast wave. Both maintain the shape of the initial perturbation, while the amplitude and polarity are decided by the initial conditions.
When a pure current perturbation is the initial condition, i.e. $\delta=0$, Eq. (2) can be simplified as

$$
\begin{aligned}
\Lambda_{1}(z, t)= & \frac{\Lambda_{0} \eta}{2} f\left(t-\frac{z}{v_{0}-c_{s}}\right)+\frac{\Lambda_{0} \eta}{2} f\left(t-\frac{z}{v_{0}+c_{s}}\right) \\
v_{1}(z, t)= & -\frac{\eta c_{s}}{2} f\left(t-\frac{z}{v_{0}-c_{s}}\right)+\frac{\eta c_{s}}{2} f\left(t-\frac{z}{v_{0}+c_{s}}\right) \\
I_{1}(z, t)= & \frac{\eta I_{0}}{2}\left[1-\frac{c_{s}}{v_{0}}\right] f\left(t-\frac{z}{v_{0}-c_{s}}\right) \\
& +\frac{\eta I_{0}}{2}\left[1+\frac{c_{s}}{v_{0}}\right] f\left(t-\frac{z}{v_{0}+c_{s}}\right) .
\end{aligned}
$$


Equation (4) indicates that energy modulations and line charge density modulations can be generated from initial modulations in beam current. Note from Eq. (4) that the fast and slow waves have the same polarity in the current profile, but the opposite sign in the energy profile. We expect the velocity and line charge density profiles to have identical amplitudes for both components. Since the current profile is the product of the two, and due to the opposite signs in the energy profile, we expect different amplitudes for the fast and slow wave when examining the current profile.

\section{EXPERIMENTAL MEASUREMENT OF SPACE-CHARGE WAVES}

In this section, we characterize space-charge waves by directly measuring both the current and the mean energy profiles. From this data, we infer the sound speed from the separation time between the peaks of opposite traveling energy or current modulations. By comparing the mean energy and current profiles, we can examine more detailed dynamics of the space-charge waves.

\section{A. The long solenoid experiment}

The long solenoid experiment (LSE) at University of Maryland [20,24-26] was designed to investigate the longitudinal dynamics of space-charge dominated beams. The apparatus consists of an electron gun [27], four short solenoids for matching, and a $1.5 \mathrm{~m}$ long solenoid. The system has been recently upgraded with additional diagnostics and improved noise shielding [28]. The upgraded system, shown in Fig. 1, now contains three Bergoz current monitors, two high-resolution energy analyzers, and a phosphor screen. The positions of the magnets and diagnostics are listed in Table I.

The two energy analyzers are inside two chambers, LC1 and LC2, before and after the long solenoid, respectively. The energy analyzers use an independently tuned focusing electrode to achieve a resolution of less than $0.2 \mathrm{eV}$ for a $5 \mathrm{keV}$ beam after considering error sources such as the device misalignment, ripples on the high voltage power supply, and data acquisition [26]. The phosphor screen in $\mathrm{LC} 1$, composed of a $\mathrm{ZnO}: \mathrm{Ga}$ deposited quartz plate with

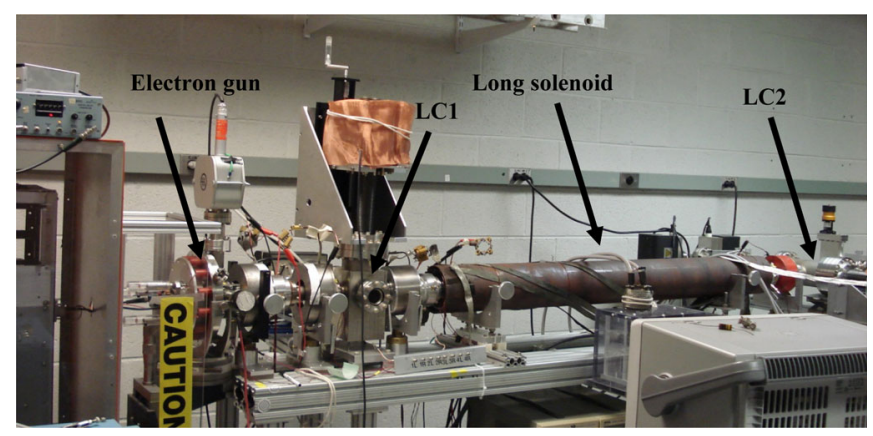

FIG. 1. (Color) A photo of the upgraded LSE system.
TABLE I. Distance in $\mathrm{cm}$ from the center of the solenoids, diagnostic chambers, and Bergoz current monitors to the downstream edge of the gun aperture.

\begin{tabular}{lccccccccc}
\hline \hline S1 & S2 & S3 & S4 & S5 & LC1 & LC2 & B1 & B2 & B3 \\
\hline 11.0 & 29.0 & 55.0 & 136.0 & 217.0 & 40.5 & 234.0 & 18.5 & 63.0 & 207.0 \\
\hline \hline
\end{tabular}

thin transparent aluminum coating, is mounted underneath the energy analyzer in the same plane as the pinhole of the analyzer, with a mirror inside the bracket at 45 degrees to deflect the beam image to the view window of chamber LC1. Aided by the $3 \mathrm{~ns}$ fast decay time of the phosphor screen, a gated intensified CCD camera can be used to obtain the time-resolved images of the beam. By moving this diagnostic complex up and down, one can use either the energy analyzer or phosphor screen to intercept the beam, providing both the mean energy profile and the transverse distribution of the beam at the same location. The Bergoz current monitors provide current profiles of the beam with a time resolution of 200 ps. To increase the signal-to-noise ratio, housings to shield each current monitor from external noise were added to the system.

\section{B. Generation of perturbations}

There are several methods for introducing perturbations on a beam: an induction module has been applied to directly modulate the beam energy [29], an optical method using the laser to create pure density perturbations [30-32], and an electronic method based on modulating the gridcathode pulse voltage of the electron gun [13]. The latter is the method we use here. Briefly, a gridded gun has a triode structure with three operating regimes: cutoff, amplification, and saturation. In saturation, the output current of the electron gun is not sensitive to the shape of the grid pulse voltage. Hence, the gun is operated in saturation mode to generate flattop beam pulse. In amplification mode, on the other hand, small fluctuations in the control grid voltage pulse are amplified and result in large variations in the output current. As a result, the amplification mode provides a way to modulate the beam current by modulating the gun pulse voltage. We note that the beam energy is also modulated by this method, except that the modulation is relatively small.

The operation mode of the electron gun is determined by the sum of two control voltages applied between the cathode and the grid: one is a DC bias voltage that forms a negative potential on the grid with respect to the cathode for suppressing emission; the other is a $100 \mathrm{~ns}$ rectangular pulse providing an opposite potential to the DC bias voltage for extracting the beam from the cathode. Hence, by adjusting the bias voltage, we can change the relative amplitude of the grid-cathode voltage during the beam pulse. The pulse voltage is formed by pulse forming line (PFL). A short cable loop can be connected to the middle 
of the PFL in parallel. As a result, a perturbation is generated at the center of the voltage pulse due to the cable loop. By connecting or disconnecting the loop, we can toggle the density modulation in the beam. Figure 2 shows a typical oscilloscope trace of the pulsed voltage signal with the applied perturbation. The amplitude of the pulsed voltage without perturbation is about $67.5 \mathrm{~V}$, and that of the perturbation is about $15 \mathrm{~V}$.

The analysis in Ref. [33] shows that the perturbation generated from this method is predominantly a current modulation rather than a velocity modulation, i.e. $|\eta| \gg$ $\delta$, where $\eta$ and $\delta$ are defined in Eq. (2). However, the contribution of initial velocity modulation does not solely rely on the comparison of $\eta$ and $\delta$ in lab frame. Since normally $v_{0}$ is much bigger than $c_{s}$, based on the $1 \mathrm{D}$ solutions in Sec. II, we introduce a dimensionless $\alpha$ factor, defined as

$$
\alpha \equiv \frac{\delta v_{0}}{\eta c_{s}}
$$

to characterize the contribution of initial velocity modulations on the amplitude of the propagating space-charge waves in lab frame [28]. When dealing with mostly current modulations, the velocity modulations can be neglected only if $\alpha$ is much less than 1 . If the ratio of $v_{0}$ and $c_{s}$ is big enough to result in a non-negligible $\alpha$, we need consider the correction by taking the initial velocity modulation into account. For example, for an electron beam with a radius of $4.9 \mathrm{~mm}$, a mean beam energy of $5075 \mathrm{eV}$, and a unperturbed current of $94.5 \mathrm{~mA}$ moving in a conducting pipe with a radius of $1.9 \mathrm{~cm}$, if the perturbation current is $7.6 \mathrm{~mA}$ and the perturbed energy is $10 \mathrm{eV}$, we can calculate $\eta=0.08, \delta=0.00097$, and $\alpha=0.167$. For this case, the value of the $\alpha$ factor is big enough to affect the amplitudes of the fast and slow waves.

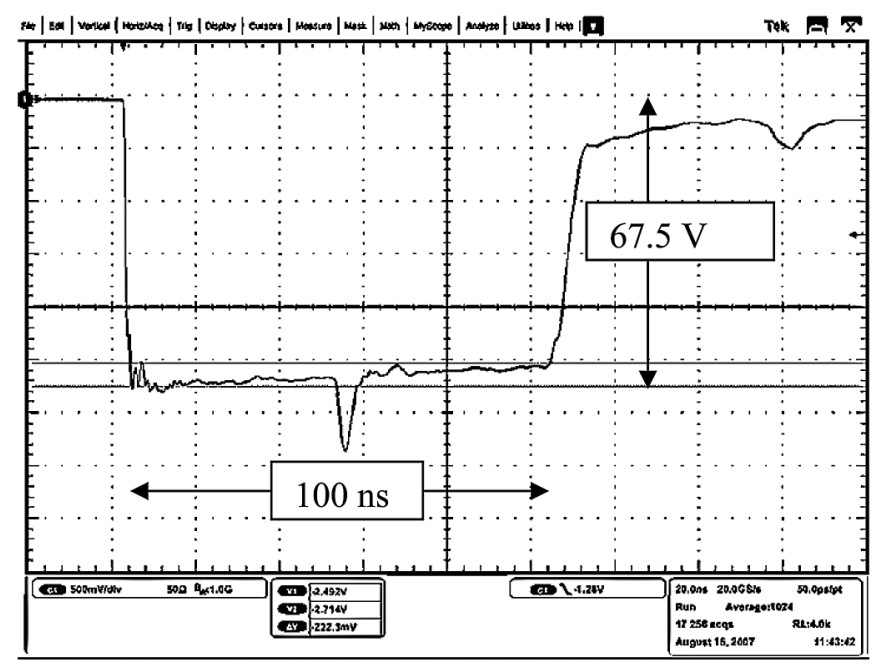

FIG. 2. The pulsed voltage signal between the grid and cathode when the perturbation cable is connected to the PFL.
When the electron gun is working in amplification mode, normally the electron beam gains more particles due to the perturbation shown in Fig. 2 and exhibits a perturbation having a similar shape. Since $\eta>0$ in this case, we call this kind of perturbation a positive perturbation. However, we found experimentally that by increasing the gap between the cathode and the anode of the gun or by applying an aperture plate near the anode plane, the beam current can be reduced under certain conditions with the same pulse voltage signal. In this case, we call it a negative perturbation throughout this paper. The mechanism of the generation of negative perturbations can be found in Ref. [33]. By connecting a low pass filter to the PFL, we can also modify the shape of the pulse voltage to be parabolic, thus generating a beam pulse with a similar shape. This "parabolic" beam has been used to test our fast imaging technique [21], and is also used in some of the simulations in Sec. V.

\section{Experimental results}

We have described how we generate both negative and positive perturbations in beam current. For all experiments described in this section, the strengths of solenoids are chosen to match the unperturbed beams into the long solenoid with a matched beam radius of $4.9 \mathrm{~mm}$. When the bias voltage was set between 30 and $35 \mathrm{~V}$, the polarities of the current perturbations were found to be negative. On the other hand, when the bias voltage was increased to $48 \mathrm{~V}$, positive perturbations were introduced to the main beam current.

Current profiles of all beams are measured by the three Bergoz current monitors (B1, B2, and B3). By comparing the total charge of different current profiles, we found that, in all cases, greater than $95.7 \%$ of the beam was transported through the whole system. The causes and consequences of the beam loss will be discussed in Sec. IV. In Figs. 3 and 4, the current profiles obtained from each of the current monitors, and the mean energy profiles at LC1 and LC2 are plotted for two cases: $V_{b}=30$ and $52 \mathrm{~V}$. The full beam length is about $100 \mathrm{~ns}$, but to emphasize the perturbed region, the beam head and tail are not shown in these figures. Unlike the current profiles in Fig. 3, where two peaks are observed only downstream of the long solenoid channel, the signatures of the fast and slow waves are already apparent in all the mean energy profiles in Fig. 4. As expected from the 1D linear cold-fluid theory, for beams with a positive initial current perturbation, the fast wave exhibits a positive pulse while the slow wave shows a negative pulse, which has already been confirmed in an earlier experiment [20]. For beams with a negative initial current perturbation, the opposite scenario takes place.

The sound speed $c_{s}$ can be derived by measuring the separation of the fast and slow waves experimentally $[28,30]$. However, if the beam transport distance is not 

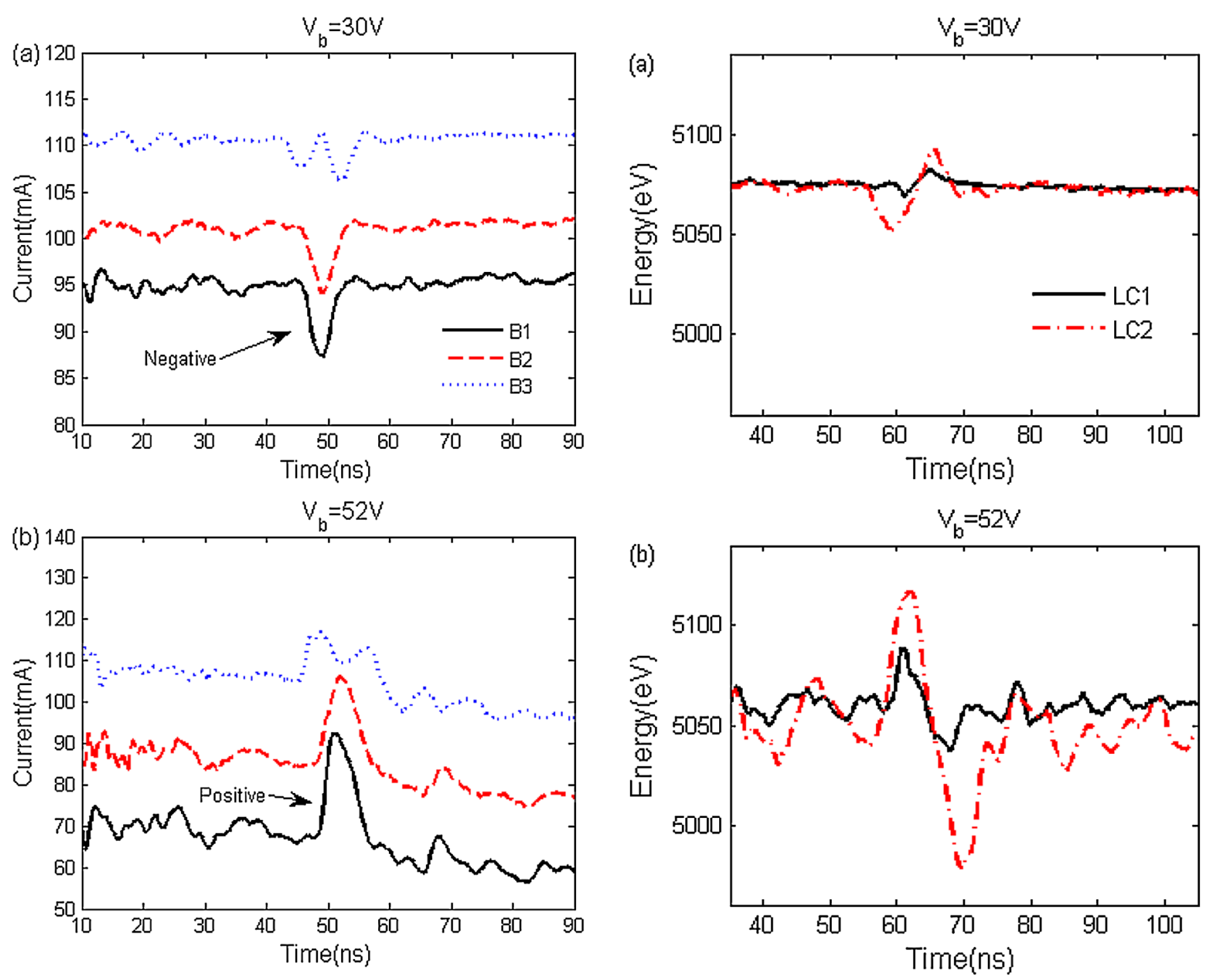

FIG. 3. (Color) Current profiles of the $4.9 \mathrm{~mm}$ beam measured by three Bergoz current monitors when the bias voltage was set to (a) $30 \mathrm{~V}$ and (b) $52 \mathrm{~V}$, respectively. In (a), current profiles measured by B2 and B3 are shifted up by 10 and $20 \mathrm{~mA}$, respectively. In (b), current profiles measured by B2 and B3 are shifted up by 20 and $40 \mathrm{~mA}$, respectively.

long enough to fully resolve the overlap of the fast and slow waves, the measurement of $c_{s}$ from the energy profile overestimates its value, while that from the current profile underestimates it (see Chap. 2 in Ref. [28]). Table II lists the calculated sound speed $c_{s}$ using Eq. (1), along with the relative errors of the measured sound speed at the location of the third Bergoz current monitor (B3) and the two diagnostic chambers (LC1 and LC2). Table II also shows some basic parameters related to these four different beams: $V_{b}$ is the bias voltage of the electron gun in each case; $I_{0}$ is the main beam current; $\eta$ is defined as the ratio of initial perturbation current $I_{1}$ to the main beam current $I_{0}$; and the main beam energy is denoted by $E_{0}$. When the perturbation strength, $\eta$, is relatively small, i.e., the cases

FIG. 4. (Color) Mean energy profiles measured at LC1 (black solid lines) and LC2 (red dashed lines) for $4.9 \mathrm{~mm}$ beams with different bias voltages. (a) $\delta_{1}=0.0026, \delta_{2}=0.0075$; (b) $\delta_{1}=0.01, \quad \delta_{2}=0.027$; where $\delta_{1}=d E_{1} / E_{0}, \quad \delta_{2}=$ $d E_{2} / E_{0}$, and $d E_{1}$ and $d E_{2}$ represent peak-to-peak values of the energy perturbations in LC1 and LC2, respectively.

for the bias voltage of 30 and $35 \mathrm{~V}$, the measured sound speeds using current profiles at the location of B3 are smaller than theoretical calculations by less than $8 \%$. However, when the perturbation strength is increased to more than $16 \%$, the measured results are more than $30 \%$ bigger than the theoretical estimates. At LC1, because the beam transport distance is too short to distinctly resolve the fast and slow waves, the measurement from energy profiles overestimates the sound speed. At LC2, the relative discrepancies are consistent for the four cases with a range from $12.5 \%$ to $15.7 \%$. However, experimental measurements underestimate the sound speed for beams with small perturbations and overestimate the sound speed for beams with larger perturbations. 
TABLE II. Parameters of current and energy profiles and calculation of sound speed.

\begin{tabular}{lccccccr}
\hline \hline & & & & & \multicolumn{2}{c}{ Relative errors of measured $c_{s}$} \\
$V_{b}(V)$ & $I_{0}(\mathrm{~mA})$ & $\eta$ & $E_{0}(\mathrm{eV})$ & $c_{s}(\mathrm{~m} / \mathrm{s})$ & At B3 & At LC1 & At LC2 \\
\hline 30.0 & 94.5 & -0.08 & 5075.0 & $2.87 \times 10^{6}$ & $-3.8 \%$ & $174.6 \%$ & $-12.5 \%$ \\
35.0 & 95.6 & -0.09 & 5070.0 & $2.88 \times 10^{6}$ & $-7.3 \%$ & $202.1 \%$ & $-15.3 \%$ \\
48.0 & 80.8 & 0.161 & 5057.0 & $2.66 \times 10^{6}$ & $32.3 \%$ & $475.2 \%$ & $14.3 \%$ \\
52.0 & 69.8 & 0.32 & 5053.0 & $2.49 \times 10^{6}$ & $34.1 \%$ & $478.5 \%$ & $15.7 \%$ \\
\hline \hline
\end{tabular}

\section{Comparison with the 1D linear cold-fluid model}

To compare the experimental results with the 1D theory, we numerically solve Eq. (2), starting with the experimentally measured current profile. In order to study the effect of different initial velocity perturbations on the evolution of fast and slow waves, for each case we assume three different initial amplitudes of the energy perturbations: 0 , 15 , and $30 \mathrm{eV}$. If $E_{1}$ represents the amplitude of the energy perturbation, the velocity perturbation strength $\delta$ can be estimated by $\delta=E_{1} /\left(2 E_{0}\right)$. Therefore, we can calculate the $\alpha$ factors using Eq. (5) for each case accordingly. With a lower bias voltage, the beam current is closer to the saturation region, hence $\alpha$ is larger as shown in Table III. Therefore, we can expect that the effect of the velocity perturbation is more significant for cases with lower bias voltages, i.e., smaller current perturbations.

Figure 5 shows beam current profiles at the location of B3 calculated from the 1D model along with the experimental results. When $V_{b}=30 \mathrm{~V}$, amplitudes of the perturbations in beam current are sensitive to the initial energy perturbations. If a zero initial energy modulation is assumed, the amplitude of the fast wave is larger than that of the slow wave, which is different from the experimental observation. The 1D results are closer to the experimental results by assuming $E_{1}=15 \mathrm{eV}$ than $E_{1}=30 \mathrm{eV}$. When $V_{b}=52 \mathrm{~V}$, the current profiles of the fast and slow waves are less sensitive to the initial condition of energy perturbations due to smaller values of the $\alpha$ factor. The two peaks in the beam current waveforms separate further in the experiment than the theoretical prediction, which is consistent with the discrepancy of sound speed between experimental measurement and theoretical calculation shown in Table II. Furthermore, we notice current loss within the perturbations in the experiment that is not present in the $1 \mathrm{D}$ results.

TABLE III. Values of $\alpha$ factor for different beams when assuming different initial energy perturbation amplitude $E_{1}$.

\begin{tabular}{lccc}
\hline \hline & $E_{1}=0$ & $E_{1}=15 \mathrm{eV}$ & $E_{1}=30 \mathrm{eV}$ \\
\hline$V_{b}=30 \mathrm{~V}$ & 0 & -0.26 & -0.52 \\
$V_{b}=35 \mathrm{~V}$ & 0 & -0.225 & -0.45 \\
$V_{b}=48 \mathrm{~V}$ & 0 & 0.135 & 0.27 \\
$V_{b}=52 \mathrm{~V}$ & 0 & 0.075 & 0.15 \\
\hline \hline
\end{tabular}

Regarding comparison of the mean energy profiles, we have obtained good agreement in a previous study [20] between experimental and 1D theoretical results for beams
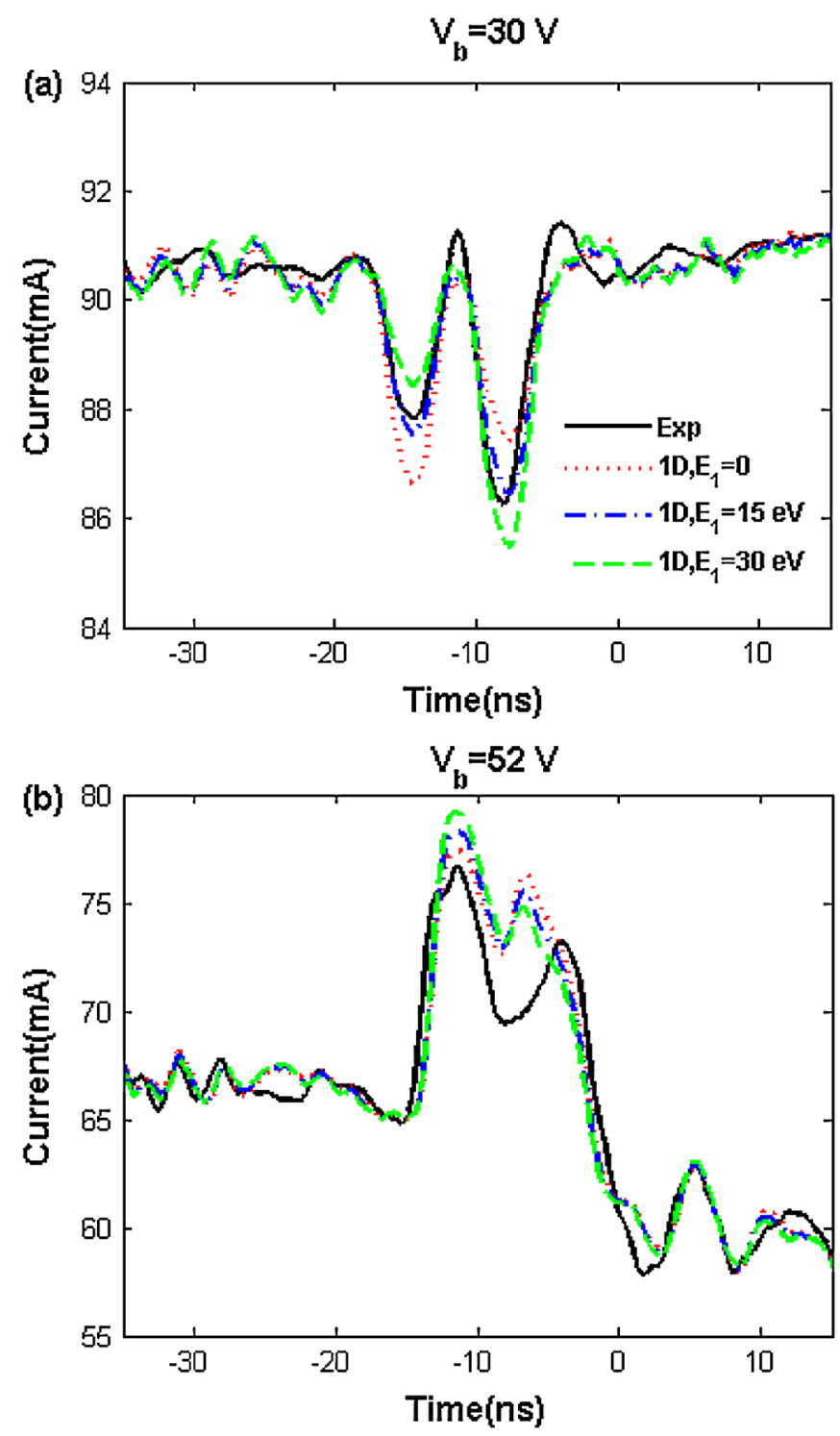

FIG. 5. (Color) Current profiles at the location of B3 calculated from 1D theory along with the experimental results (black solid lines). In the 1D theory, the amplitude of initial energy perturbation is assumed to be 0,15 , and $30 \mathrm{eV}$, respectively. The beam currents are represented by positive values. 

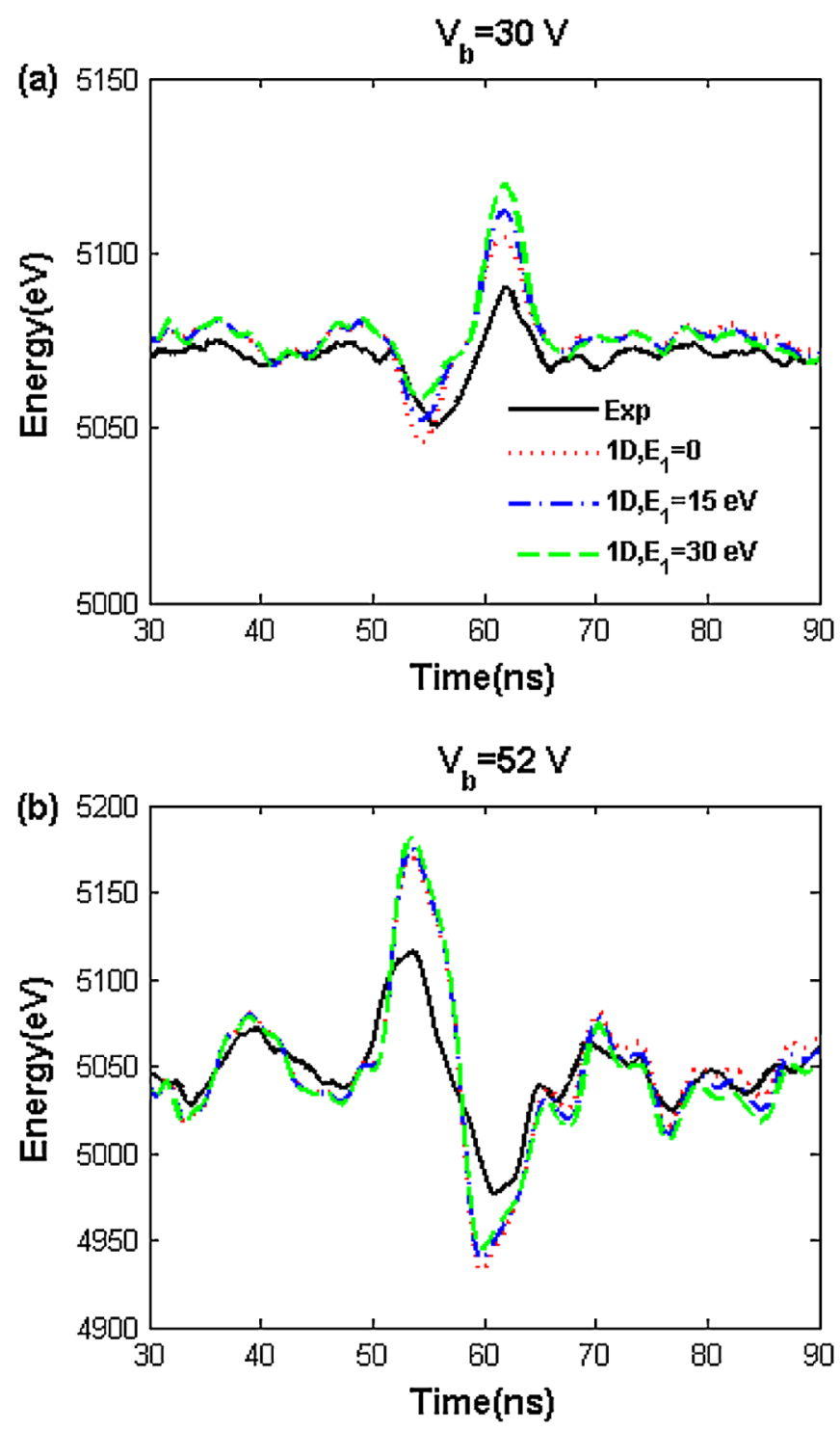

FIG. 6. (Color) Energy profiles at LC2 calculated from 1D theory along with the experimental results (black solid lines). In the $1 \mathrm{D}$ theory, the amplitude of initial energy perturbation is assumed to be 0,15 , and $30 \mathrm{eV}$, respectively.

with small perturbations. However, as shown in Fig. 6 for this experiment, discrepancies remain in the mean energy profiles at LC2 for both the separation and amplitudes of the two peaks, even for the small-amplitude case. Several reasons may contribute to these discrepancies: first, it is possible that the relatively large beam size in the matching section of the transport line reduces the $g$ factor leading to both slower propagation and smaller perturbation amplitudes of the space-charge waves; second, some other mechanism, such as beam loss may limit the perturbation strength growth; finally, the unknown temporal behaviors of beams inside the energy analyzers affect the coherent errors when measuring a beam with energy modulations, a subject deserving further study.

\section{SIMULATION STUDIES OF SPACE-CHARGE WAVES}

As described in Sec. II, the 1D cold-fluid model assumes a monoenergetic distribution of the unperturbed beam, thus the kinetic effects are missed in the linear theory for small perturbations. However, kinetic effects can be described by the Vlasov equation if we neglect collisionality. The PIC model in WARP is a method for integrating the Vlasov equation using macroparticles to discretize the distribution function. In this section, we apply the particle-in-cell code WARP to model the experiment, using the experimental data upstream around LC1 to initialize the simulation, and compare the output to the experimental data downstream at and near LC2. Initial conditions such as density and velocity distributions of macroparticles need to be specified. In addition, to calculate the field distribution, grids need to be defined in space. On each grid point, the selfconsistent electric and magnetic fields are calculated from the distribution of macroparticles and boundary conditions. The macroparticles are pushed by the fields, interpolated from the self-consistent fields on the neighboring grid points, to new positions with an updated distribution. Then the field calculations and particle pushing are iterated each time step. WARP has the capability of simulating the beam in either $R-Z$ or full 3D geometry.

To simplify the presentation, we concentrate here on two perturbed cases, one with a particle increase and one with a particle decrease. In addition, we introduce a third case where the beam current profile is roughly parabolic. Experimental measurements on all three cases have been presented earlier in Ref. [21].

\section{A. The WARP simulation model and tests on a parabolic beam}

To calibrate the simulation model, we start with a parabolic beam having a peak current of about $23 \mathrm{~mA}$, which has been used to test the fast phosphor screen [21]. First we simulated the transport of the parabolic beam in the LSE system using WARP-RZ code in beam frame. We assume uniform focusing to represent the long solenoid with focusing strength $\kappa=70.5 \mathrm{~m}^{-2}$. The current profile measured by B 1 was imported into WARP as the initial condition of the beam current [21]. For the beam-frame simulations, we scale the time axis of the measured current profile by the main beam velocity to get a current profile as a function of position. Transversely, we use an initially semiGaussian distribution, which is uniform in space and Gaussian in velocity space, with a uniform temperature. We assume a constant initial radius of $3.5 \mathrm{~mm}$ (corresponding to the matched radius of a $14 \mathrm{~mA}$ beam inside the long solenoid), a zero slope, and a constant longitudinal kinetic energy of $5050 \mathrm{eV}$ along the beam. Numerical settings for the simulation have been systematically varied to assure convergence of the simulations. Converged parameters for the beams used are: 200000 macroparticles; a time step of 
$0.6 \mathrm{~ns} ; 32$ cells in each of the transverse $x$ and $y$ directions (or in the radial direction for $R-Z$ simulations); and 256 cells in the $z$ direction. The resulting grid spacing is $0.595 \mathrm{~mm}$ in the $x$ or $y$ direction and $8.24 \mathrm{~mm}$ in the $z$ direction.

We extracted the mean beam energy profiles from the simulation when the beam center was transported for 40.5 and $234.0 \mathrm{~cm}$, i.e., the distance from the gun aperture plate to the front plates of two energy analyzers in the LSE system. These are compared in Fig. 7 with the energy profiles measured experimentally using the energy analyzers. One should note that the vertical scales of Figs. 7(a) and 7(b) are different. In LC1, the simulation result has a good fit with the experimental measurement for the center of the beam, namely from 30 to $50 \mathrm{~ns}$, but shows higher energy for the head and lower energy for the tail than those measured in the experiment. In addition, the mean energy profile from the simulation features more oscillations than the experimental results. This difference between the experiment and the beam-frame simulation is amplified by the time the beam reaches LC2. The simulation also exhibits a larger energy tilt at the beam center due to the fact that the beam head travels a longer distance in the simulation than the tail.

To obtain a more accurate comparison with the experiment, we performed WARP-RZ simulations for the same beam in the lab frame. Here, the simulation starts from the front surface of the energy analyzer in LC1 instead of the gun aperture, and a number of particles are injected at each time step proportional to the beam current measured in B1. The transverse radius and the energy of the injected particles are adjusted to values measured by the fast phosphor screen and the energy analyzer at LC1. All these measured profiles can be found in Ref. [21]. The beam slope and emittance had not been measured at that plane as a function of time, so we assumed a zero slope and a constant emittance of $40 \mu \mathrm{m}$ initially. The time step in the simulation is $20.9 \mathrm{ps}$. We inject 40 macroparticles per $\mathrm{mA}$ each step, resulting in about $1.5 \times 10^{6}$ for the whole simulation. In addition, we included the full $z$ dependence of the solenoid fields, including nonlinearities up to 5th order. The solenoids were modeled by calculating the magnetic fields on a 3D grid with a $1 \mathrm{~mm}$ resolution using an expansion from the measured axial field profiles. In order to minimize the effect of the boundary on the beam at the end of the transport line, we transport the beam $31 \mathrm{~cm}$ beyond the front plate of the second energy analyzer before it hits the boundary, which is periodic for fields and absorbing for particles. Thus, in order to focus the beam in this short distance, we added a solenoid identical to S5 in the simulation. This solenoid, S6, will not affect the simulation results because it is placed $12 \mathrm{~cm}$ away from the energy analyzer in LC2, which is a relatively large distance compared to its effective length shown in Table I. We increased the number of grid cells in the $z$ direction to
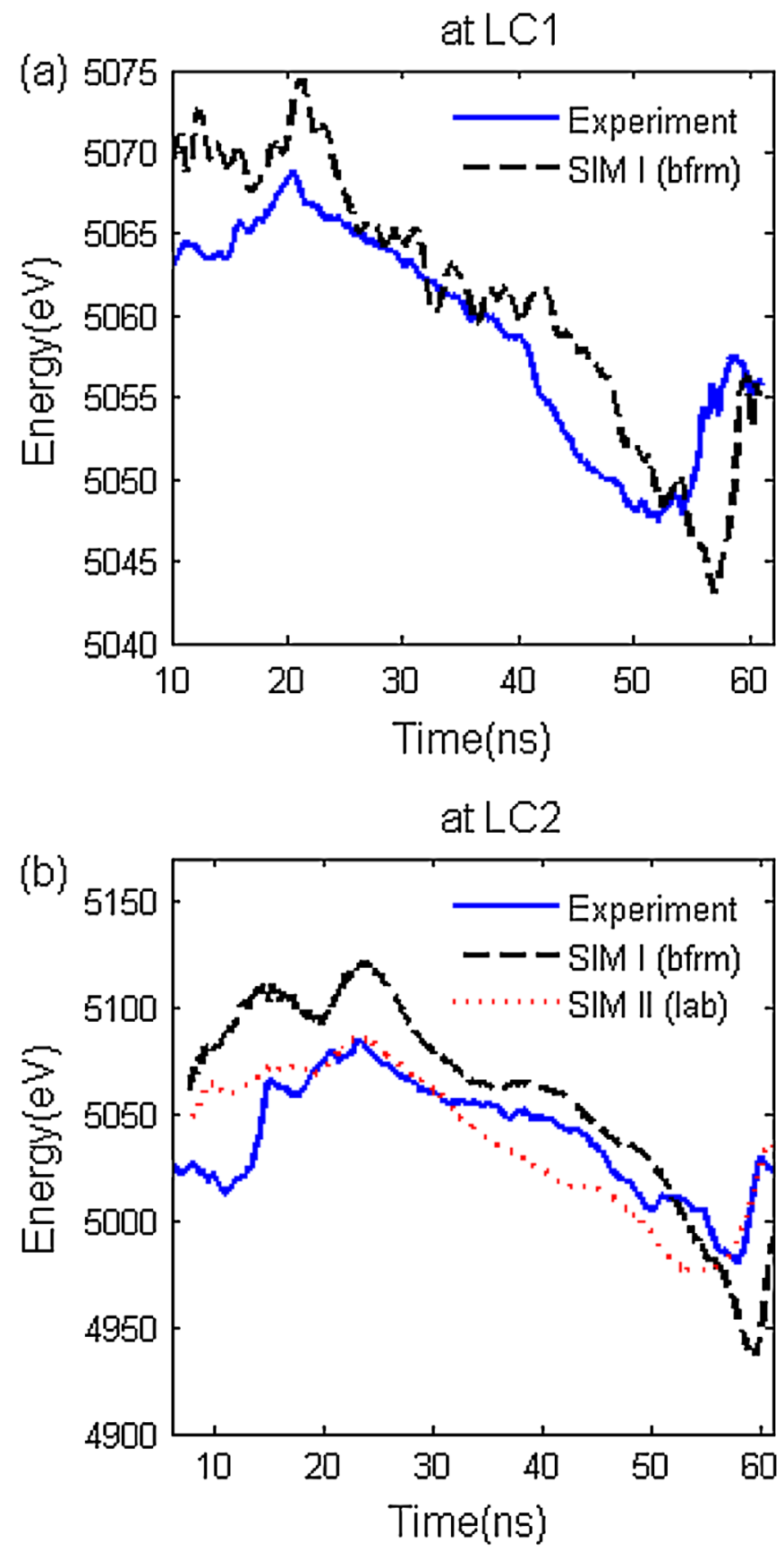

FIG. 7. (Color) Comparison of mean energy profiles of the $23 \mathrm{~mA}$ parabolic beam between the experimental results, WARP simulations in the beam frame and lab frame: (a) at LC1; (b) at LC2.

2048, corresponding to a grid spacing of $1.10 \mathrm{~mm}$. Remaining numerical parameters are the same as mentioned earlier.

The lab-frame simulation result is plotted as the dotted red line in Fig. 7(b). It shows that, using the simulation in the lab frame, we achieve closer agreement with the experimental measurement of the mean energy profile. However, some discrepancy still exists. For example, the measured mean energy profile at the beam center is not as 
linear as that predicted by the simulation. This suggests that the longitudinal electric fields calculated in the simulation are more linear than those acting on the beam in experiment. Since the longitudinal electric field $E_{z}$ is related to the derivative of line charge density, we next examine the current profiles and find that the current profiles from the WARP simulation are almost identical at different locations from $\mathrm{LC} 1$ to $\mathrm{B} 3$. The preservation of current profiles during the beam transport implies an invariance of $E_{z}$. On the contrary, we observed some beam loss in the experiment at B3, especially in the middle of the bunch where the beam is largest transversely. As a result, the longitudinal self-electric field is expected to change and becomes modulated.

\section{B. Simulations of rectangular beams with perturbations}

Having calibrated the simulations using a relatively simple beam profile, and thus determined the relevant simulation parameters, we apply in this section the labframe WARP model to the two rectangular perturbed beams described in Ref. [21], which have a main beam current of 25 and $40 \mathrm{~mA}$, respectively. One should note that these beams are distinct from those presented in Sec. III. As discussed in the previous section, the transport channel in WARP consists of four solenoids starting from LC1. The strengths of the solenoids are set to be consistent with those used in experiments. Since the perturbations for both beams are less than $10 \mathrm{~ns}$ wide and the longitudinal shapes of the unperturbed beams are rectangular, we inject only a part of the beam around the perturbation in order to reduce the computation time. Longer beams of $60 \mathrm{~ns}$ have also been tested to ensure that the truncation does not affect the simulation results. We compare the beam current profiles and mean energy profiles obtained from simulations with experimental data and analytical results from 1D calculation for both cases. For both beams to be described, we use the same numerical settings as those in the lab frame simulations of the parabolic beam. The initial transverse beam size, longitudinal current, and mean energy profiles are injected by importing the measured data.

\section{Simulation of the $25 \mathrm{~mA}$ beam (small-amplitude negative perturbation)}

For the $25 \mathrm{~mA}$ perturbed beam, with an initial perturbation current of about $5 \mathrm{~mA}$, we simulate $38 \mathrm{~ns}$ of the beam bunch centered around the perturbation. In Fig. 8, we present both mean energy profiles at LC2 and current profiles at B3 obtained from the experiment, WARP simulation, and 1D cold-fluid theory, respectively. We assume the following beam parameters in the fluid calculation: a radius of $2.7 \mathrm{~mm}$; nominal beam energy of $5050 \mathrm{eV}$; an unperturbed main beam current of $25 \mathrm{~mA}$; an initial current perturbation strength $\eta$ of -0.2 ; an initial energy pertur-
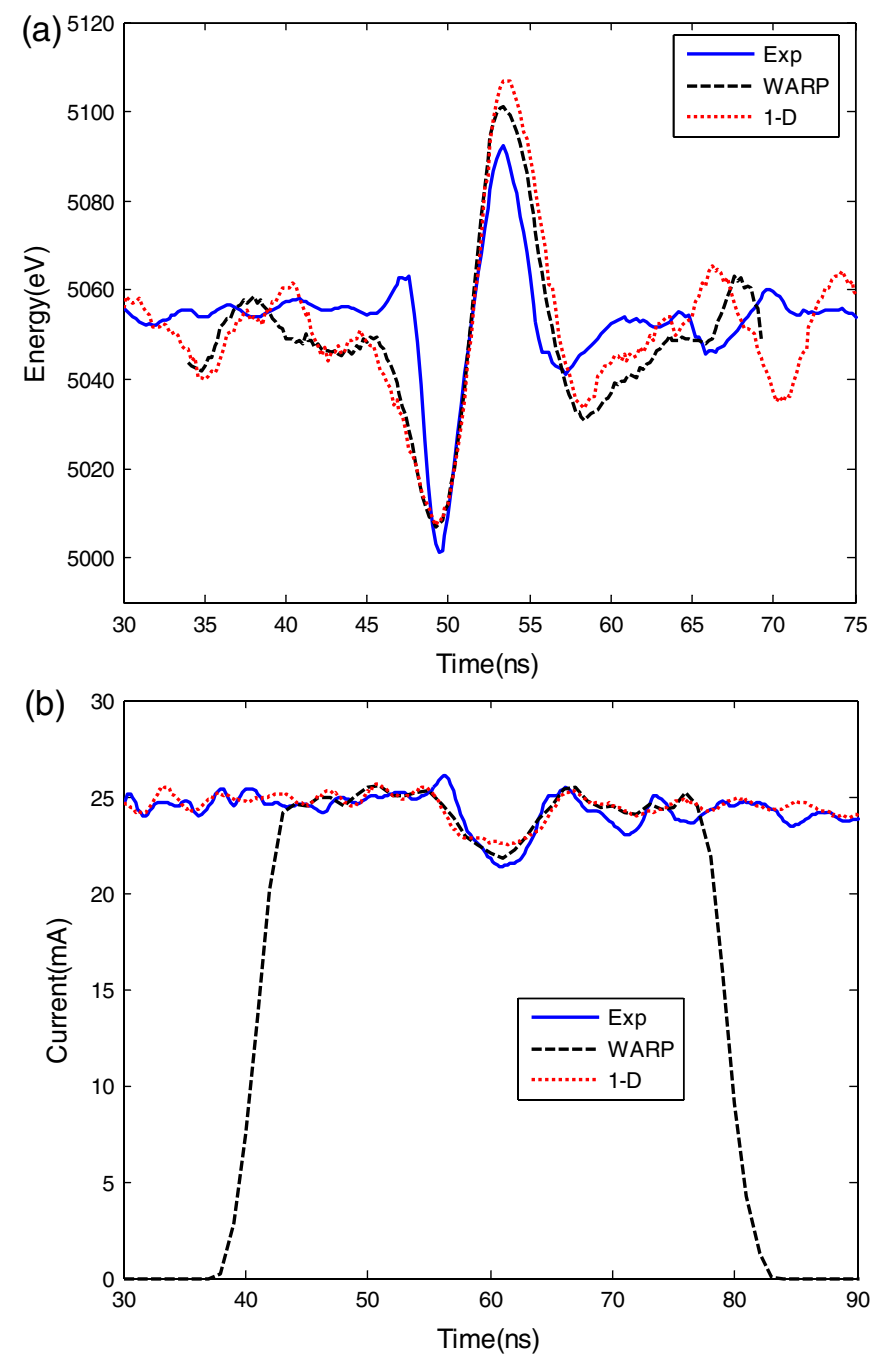

FIG. 8. (Color) Comparison of results from the experiment (blue solid lines), WARP (black broken lines), and 1D theory (red dotted lines) for the $25 \mathrm{~mA}$ beam: (a) mean energy profiles at LC2; (b) current profiles at B3.

bation of $10 \mathrm{eV}$. Hence, we can derive the sound speed $c_{s}=1.87 \times 10^{6} \mathrm{~m} / \mathrm{s}$ and $\alpha=-0.11$.

In the mean energy profiles, the peak-to-peak amplitudes of the energy modulations in LC2 are close for all cases, namely 90, 94, and $100 \mathrm{eV}$ for the results from the experiment, WARP, and theory, respectively. The separation time between the two peaks of the energy modulations are also consistent with the difference of the peak-to-peak amplitudes: the 1D result has the largest separation and the experimental measurement shows the smallest. These suggest that the experiment measures a smaller sound speed than predictions from both WARP and 1D theory.

The current profile from the 1D model exhibits the start of separation of the fast and slow waves, but both results from WARP and the experiment indicate a single negative peak in the current waveforms. Also, the amplitude of the experimental results is the largest among these three traces. When the two wave components are moving apart in the 
current profile, their sum will first exhibit a single peak with smaller amplitude and wider shape before developing into two clear peaks. Thus, the comparison in Fig. 8(b) leads to the same conclusion as that in Fig. 8(a): the average sound speed $c_{s}$ in experiment is smaller than the theoretical prediction and numerical simulation. However, the result from the WARP simulation is closer to the experimental results than that from the 1D model, indicating the effect of ignoring nonlinear terms in the fluid equations.

To test the sensitivity to initial conditions, we ran additional WARP simulations with different initial data and found that the simulation results were not sensitive to initial beam radius, energy, and emittance. The physics of the longitudinal space-charge waves governing the WARP simulations is still consistent with the $1 \mathrm{D}$ cold-fluid theory. In other words, the longitudinal and transverse dynamics are still simply correlated through the $g$ factor.

\section{Simulation of the $40 \mathrm{~mA}$ beam (large-amplitude positive perturbation)}

For the $40 \mathrm{~mA}$ beam, which has a peak perturbation current of $47.6 \mathrm{~mA}$, we only inject $40 \mathrm{~ns}$ of the beam bunch. For the fluid calculations, the beam radius is $4.3 \mathrm{~mm}$; the nominal beam energy is $5050 \mathrm{eV}$; the unperturbed main beam current is $40 \mathrm{~mA}$; the initial current perturbation strength $\eta$ is 1.19 ; the initial energy perturbation is $10 \mathrm{eV}$. Hence, we can derive the sound speed $c_{s}=$ $2.07 \times 10^{6} \mathrm{~m} / \mathrm{s}$ and $\alpha=0.017$.

Figure 9 shows large discrepancies in this case between the experiment, WARP, and theory. The 1D calculation and WARP simulation predict a peak-to-peak energy modulation of 630 and $541 \mathrm{eV}$, respectively, corresponding to $12.5 \%$ and $10.7 \%$ of the main beam energy. However, in the experiment, we obtained a much smaller energy modulation with a peak-to-peak amplitude of $309 \mathrm{eV}$. The shapes of the fast and slow waves are not preserved in the experiment. Because the approximation of small perturbations is not applicable to this beam, the discrepancy between experiment and 1D theory is expected. The WARP code, however, does not make any linear approximation; therefore its inconsistent result with measurement suggests a mechanism in the experiment which prevents the energy modulation from reaching its full extent.

The comparison of current profiles shown in Fig. 9(b) indicates that beam loss occurs in the experiment at the location of the perturbation. Further calculations show that the perturbation loses approximately $20 \%$ of its total charge. As discussed earlier for the parabolic beam, this may be a reason for the observed discrepancy in mean energy profiles. Comparing WARP with the theory gives an indication of the magnitude of the nonlinear effects neglected by the theory. We note that, while the mean energy profiles are different, the current profiles also differ in detailed structure, despite having similar amplitudes and widths. This suggests that the nonlinearity of space-charge
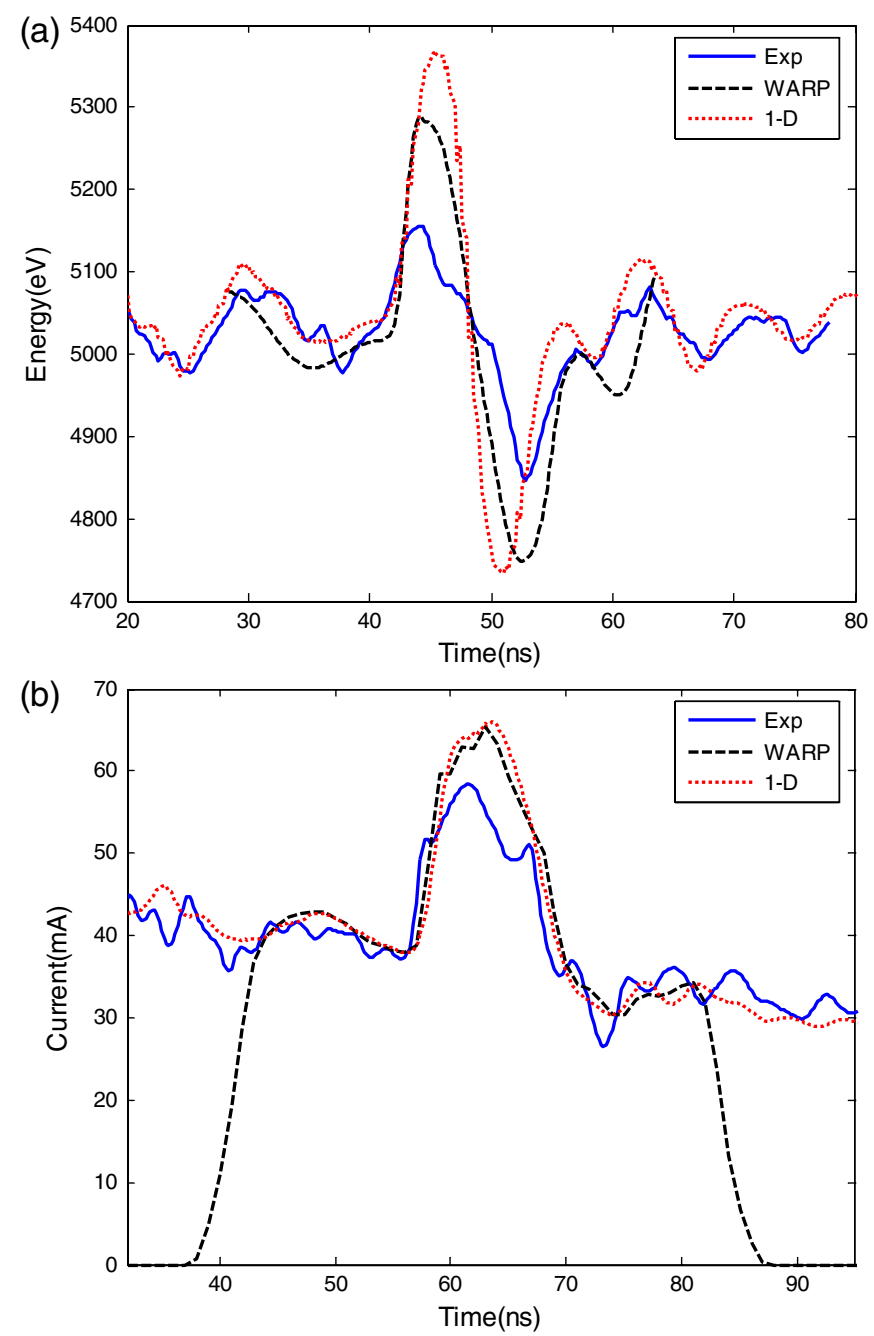

FIG. 9. (Color) Comparison of results from the experiment (blue solid lines), WARP (black broken lines), and 1D theory (red dotted lines) for the $40 \mathrm{~mA}$ beam: (a) mean energy profiles at LC2; (b) current profiles at B3. Current profiles have been flipped to show positive current values for the convenience of indicating positive perturbations.

waves in this case affects the mean energy profile more than it does the current profile.

To investigate the effect of beam loss on the longitudinal beam energy profile, we ran additional WARP simulations in which we artificially scraped the beam by applying virtual conducting tubes with different radii, concentric with the beam pipe. This tube is virtual in the sense that it is only used to remove particles wandering beyond the wall radius without affecting the self-field calculations. Figure 10 indicates two different wall radii that were used: 8 and $9 \mathrm{~mm}$. As our simulation is in $R-Z$ geometry, the projection of the particle distribution in $X-Z$ plane is equivalent to that in the $R-Z$ plane. The current and mean energy profiles from these simulations are presented in Fig. 11, where $r_{w}$ represents the radius of the virtual tube. When $r_{w}=1.9 \mathrm{~cm}$, the virtual tube has the same radius as the beam pipe, the 


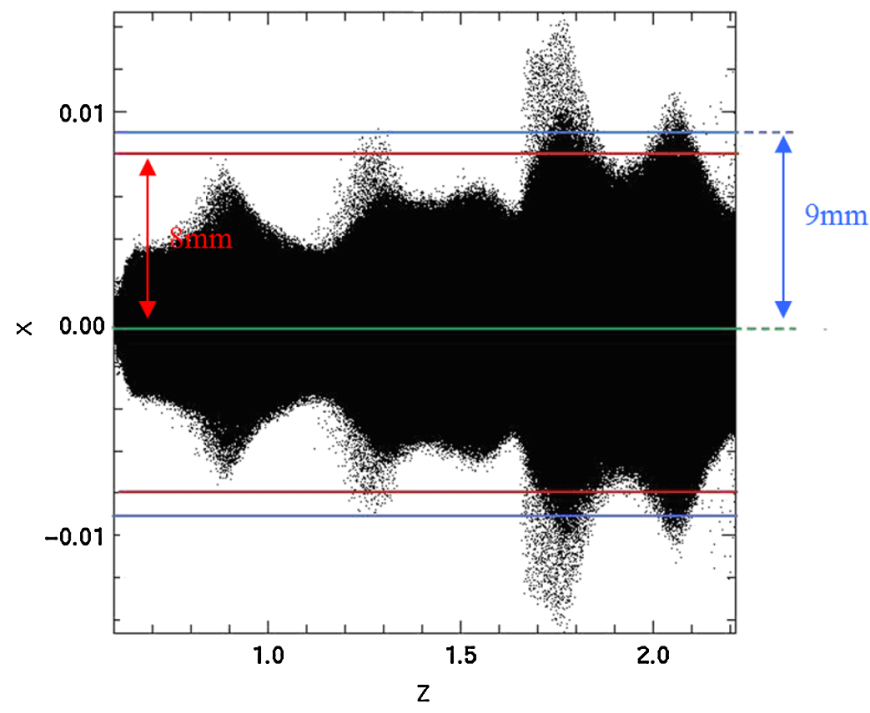

FIG. 10. (Color) Illustration of applying two virtual conducting tubes in WARP code and the particle distribution in the $X-Z$ plane at $t=57.2 \mathrm{~ns}$. The units of both axes are meter. The perturbation is located from 1.22 to $1.7 \mathrm{~m}$.

same result presented earlier. The current profile is closest to the experimental result when $r_{w}=9 \mathrm{~mm}$. For that scraping radius, the mean energy profile, while closer to the experimental result than the unscraped beam, still has a significant discrepancy. If the radius of the virtual tube is further reduced to $8 \mathrm{~mm}$, the mean energy profile becomes closer to the experimental result, but the current loss exceeds that of the experiment. Hence, the results in Fig. 11 suggest that the discrepancy between simulation and experiment can be caused by the scraping of the beam due to misalignment or mismatch, but there is a phase difference between mean energy and current profiles. To investigate how a different combination of mismatch could adjust this phase difference, we conducted a series of simulations using the $R-Z$ code with a $9 \mathrm{~mm}$ virtual tube, as in Fig. 11, but changing the initial beam radius to mimic a mismatch. The results of such a simulation, with the initial beam radius at $4 \mathrm{~mm}$, are plotted as the red dash-dotted lines in Fig. 12, demonstrating closer agreement with the experiment.

The introduction of a virtual conductor is a somewhat artificial way of modeling mismatch and misalignments in an $R-Z$ geometry. The question remains whether such errors in the experiment that can decrease the aperture by $10 \mathrm{~mm}$ are reasonable. Examination of solenoid scan data using the phosphor screen in $\mathrm{LC} 1$ reveals beam movement of the order of $1 \mathrm{~cm}$ depending on the current and polarity of solenoid S2. This is likely due to a misalignment of the electron gun relative to the magnetic axis of the solenoids. From this data, we conclude that a misalignment of the beam centroid of the order of $5 \mathrm{~mm}$ or more is likely, although it is difficult to pin down the exact initial conditions with the scan of one solenoid. We have further

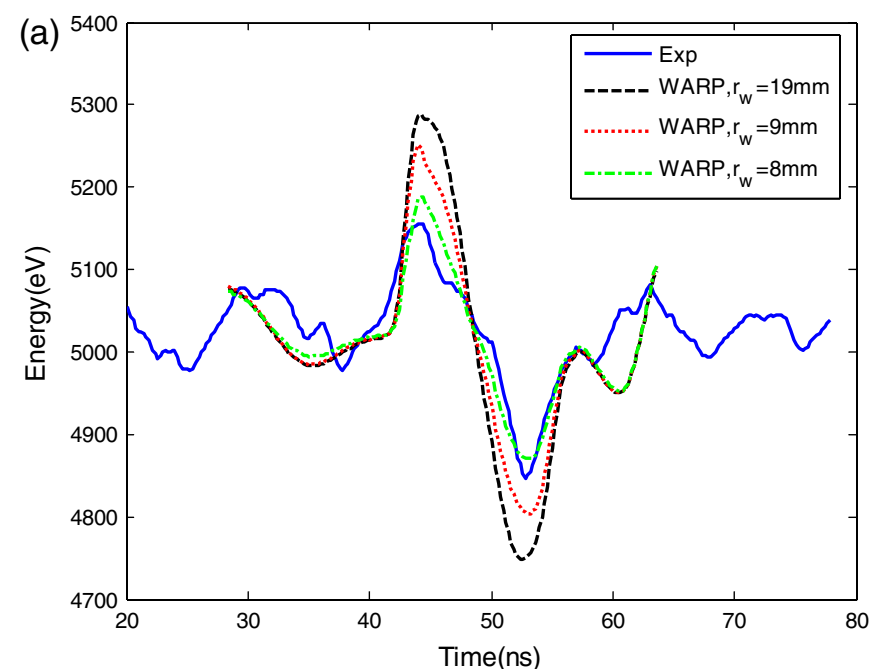

(b)

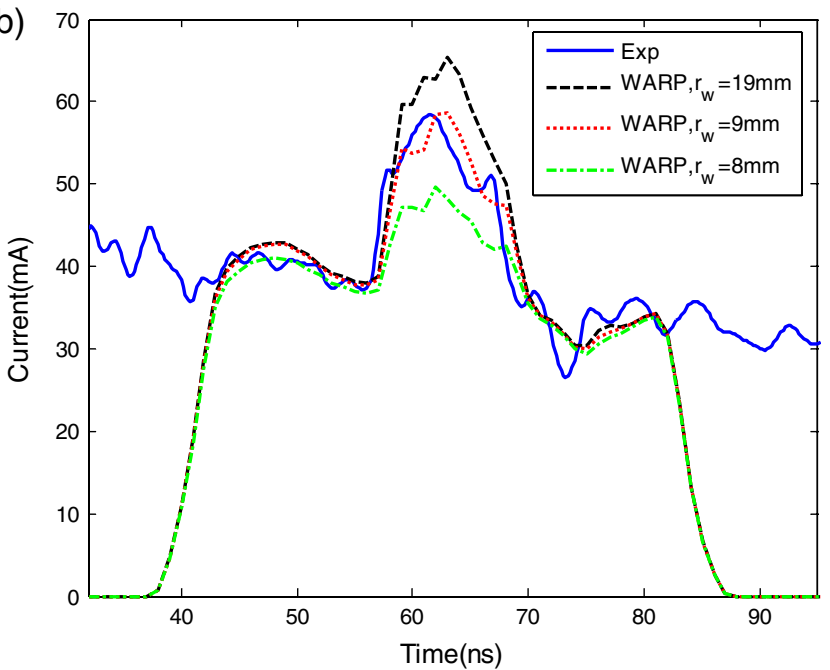

FIG. 11. (Color) Comparison of results from the experiment (blue solid lines), WARP simulations with different radii of the virtual tube: $19 \mathrm{~mm}$ (black dashed lines), $9 \mathrm{~mm}$ (red dotted lines), and $8 \mathrm{~mm}$ (green dash-dotted lines): (a) mean energy profiles at LC2; (b) current profiles at B3. Current profiles have been flipped to show positive current values for the convenience of indicating positive perturbations.

tested this hypothesis using 3D lab-frame WARP simulations in which we introduce an initial misalignment of the beam centroid, keeping the scraping radius equal to the pipe radius at $19 \mathrm{~mm}$. Figure 12 (green dotted lines) shows the results of a 3D simulation in which the beam has an initial radius of $4 \mathrm{~mm}$ and a slope of $0.05 \mathrm{rad}$, and is offset by $5 \mathrm{~mm}$. The 3D simulation qualitatively replicates the earlier agreement of the $R-Z$ simulation, but a disagreement in energy profiles remains.

Because of the lack of a theoretical model for the evolution of large-amplitude perturbations, it is difficult to judge how much the beam loss and mismatch contributes to the discrepancies shown in Fig. 9. Other factors, such as the transient behavior of the energy analyzer and the longitudinal-transverse coupling, should also be investigated in future studies. 

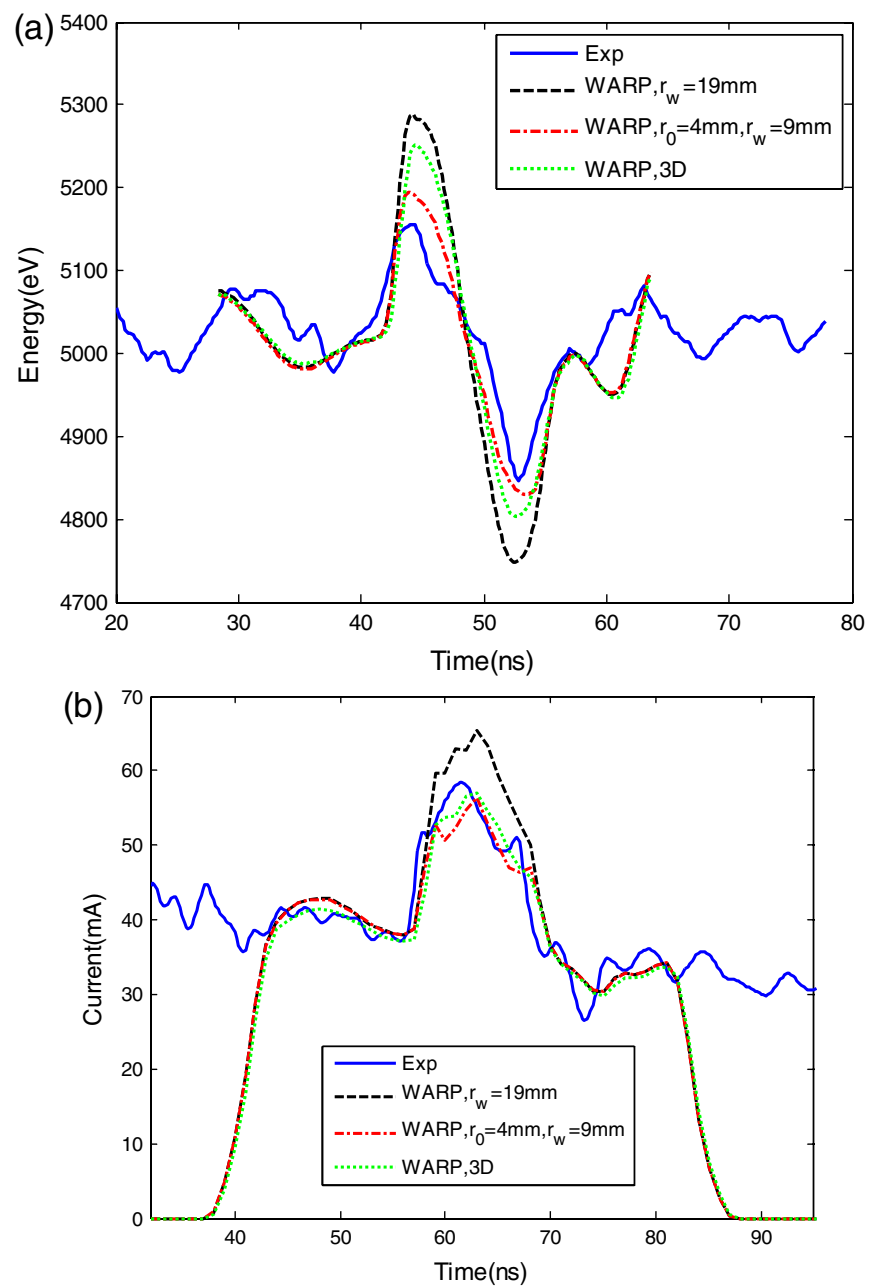

FIG. 12. (Color) Comparison of results from the experiment (blue solid lines), WARP simulations with $19 \mathrm{~mm}$ virtual tube (black dashed lines), WARP simulations with the $9 \mathrm{~mm}$ virtual tube and the initial beam radius of $4 \mathrm{~mm}$ (red dash-dotted lines), and WARP 3D simulations (green dotted lines): (a) mean energy profiles at LC2; (b) current profiles at B3.

\section{CONCLUSIONS}

We have presented detailed experimental measurements on the LSE of both the current and mean energy profiles for beams with initial perturbations in current. By comparing the experimental results with analytical solutions from a 1D cold-fluid model, we have verified the applicability of the theory for predicting general trends of the evolution of space-charge waves. In addition, we also found that, in spite of its small amplitude, the initial velocity modulation usually cannot be neglected for beams with small current perturbations.

The results from the lab-frame simulation in WARP show good agreement with experimental results for both the parabolic beam and the $25 \mathrm{~mA}$ rectangular beam with a $20 \%$ perturbation. For the beam with an extremely large perturbation, the discrepancy between WARP and 1D theory can be attributed to the linear assumption made in deriving the theory. However, the large deviation of the experimental measurement from the simulation was unexpected. Although the WARP simulations presented do not have sufficiently accurate initial conditions to reproduce the experiment in detail, they clearly suggest that the beam loss due to mismatch or misalignment can significantly modify the mean energy and current profiles, thus contributing to the inconsistency between experiment and simulation. Hence, the results presented in this paper also suggest that special attention should be paid to the possible beam loss caused by mismatch during the operation of machines with high intensity beams, such as acceleratordriven heavy ion fusion and the new generation of electron light sources.

Recently, by employing tomography, we have developed a time-resolved phase space measurement technique with a resolution of 3 ns $[34,35]$. It is possible, by combining this information with the longitudinal phase space measured by the energy analyzer, to map the time-resolved full 6D phase space of a space-charge dominated beam. In the future, this $6 \mathrm{D}$ phase space can be fed to a 3D lab-frame simulation to produce more accurate results. They can help us simulate the mismatch of the large-amplitude perturbation more accurately, and test sensitivity to misalignment. They may also provide some clues about the beam loss and its relationship with the $g$ factor.

\section{ACKNOWLEDGMENTS}

We thank Y. Zou, Y. Cui, M. Walter, S. Bernal, B.L. Beaudoin, D. Sutter, D. Cohen, D. Stratakis, D. Feldman, R. Fiorito, and C. Wu for helpful discussions. We also thank A. Friedman, D. P. Grote, and J.-L. Vay for providing the WARP code. This work is supported by the U.S. Department of Energy High Energy Physics and Fusion Energy Science, and by the Department of Defense Office of Naval Research and Joint Technology Office.

[1] D. H. Dowell and P. G. O'Shea, in Proceedings of the 1997 Particle Accelerator Conference, Vancouver, BC (IEEE, New York, 1998), p. 1891.

[2] K. Bane et al., in Proceedings of the 2007 Particle Accelerator Conference, Albuquerque, New Mexico (IEEE, Albuquerque, New Mexico, 2007), p. 807.

[3] H. H. Braun, R. Corsini, L. Groening, F. Zhou, A. Kabel, T. O. Raubenheimer, R. Li, and T. Limbert, Phys. Rev. ST Accel. Beams 3, 124402 (2000).

[4] G. Stupakov and S. Heifets, Phys. Rev. ST Accel. Beams 5, 054402 (2002).

[5] S. Reiche and J. B. Rosenzweig, Phys. Rev. ST Accel. Beams 6, 040702 (2003).

[6] Z. Huang, M. Borland, P. Emma, J. Wu, C. Limborg, G. Stupakov, and J. Welch, Phys. Rev. ST Accel. Beams 7, 074401 (2004). 
[7] A. Friedman et al., in Proceedings of the 2005 Particle Accelerator Conference, Knoxville, TN (IEEE, New York, 2005), p. 339.

[8] R. F. Welton, M. P. Stockli, and S. N. Murray, Rev. Sci. Instrum. 75, 1793 (2004).

[9] C. A. Brau, Free-Electron Lasers (Academic Press, San Diego, 1990).

[10] P. A. Seidl et al., in Proceedings of the 2003 IEEE Particle Accelerator Conference, Portland, OR (IEEE, New York, 2003), p. 536.

[11] F. M. Bieniosek et al., Nucl. Instrum. Methods Phys. Res., Sect. A 544, 268 (2005).

[12] E.P. Gilson, R.C. Davidson, P.C. Efthimion, and R. Majeski, Phys. Rev. Lett. 92, 155002 (2004).

[13] J. G. Wang et al., Phys. Rev. Lett. 71, 1836 (1993).

[14] M. Reiser, Theory and Design of Charged Particle Beams (John Wiley \& Sons, Inc., New York, 1994).

[15] D. X. Wang, J. G. Wang, and M. Reiser, Phys. Rev. Lett. 73, 66 (1994).

[16] Y. Cui, Y. Zou, A. Valfells, M. Reiser, I. Haber, R. A. Kishek, S. Bernal, and P. G. O'Shea, Rev. Sci. Instrum. 75, 2736 (2004).

[17] Y. Zou, Y. Cui, I. Haber, M. Reiser, and P. G. O'Shea, Phys. Rev. ST Accel. Beams 6, 112801 (2003).

[18] H. Suk et al., J. Appl. Phys. 86, 1699 (1999).

[19] Y. Zou, J. G. Wang, H. Suk, and M. Reiser, Phys. Rev. Lett. 84, 5138 (2000).

[20] K. Tian, Y. Zou, Y. Cui, I. Haber, R. A. Kishek, M. Reiser, and P. G. O'Shea, Phys. Rev. ST Accel. Beams 9, 014201 (2006).

[21] K. Tian, R. A. Kishek, P. G. O'Shea, R. B. Fiorito, D. W. Feldman, and M. Reiser, Phys. Plasmas 15, 056707 (2008).
[22] D. P. Grote, A. Friedman, I. Haber, and S. Yu, Fusion Eng. Des. 32-33, 193 (1996).

[23] J. R. Harris, Doctoral dissertation, University of Maryland, 2005.

[24] Y. Zou, Y. Cui, M. Reiser, and P. G. O’Shea, Phys. Rev. Lett. 94, 134801 (2005).

[25] Y. Cui, Doctoral dissertation, University of Maryland, 2004.

[26] Y. Cui, Y. Zou, M. Reiser, R. A. Kishek, I. Haber, S. Bernal, and P. G. O'Shea, Nucl. Instrum. Methods Phys. Res., Sect. A 544, 527 (2005).

[27] J. G. Wang et al., IEEE Trans. Electron Devices 37, 2622 (1990).

[28] K. Tian, Doctoral dissertation, University of Maryland, 2008.

[29] B. L. Beaudoin, S. Bernal, I. Haber, R. A. Kishek, P. G. O'Shea, M. Reiser, J. C. T. Thangaraj, K. Tian, M. Walter, and C. Wu, in Proceedings of the 2007 Particle Accelerator Conference, Albuquerque, New Mexico (Ref. [2]), p. 2322.

[30] Y. Huo, Master thesis, University of Maryland, 2004.

[31] J.C.T. Thangaraj et al., in Proceedings of the 2007 Particle Accelerator Conference, Albuquerque, New Mexico (Ref. [2]), p. 3570.

[32] J. R. Harris, J. G. Neumann, and P. G. O'Shea, J. Appl. Phys. 99, 093306 (2006).

[33] J. R. Harris and P. G. O'Shea, IEEE Trans. Electron Devices 53, 2824 (2006).

[34] D. Stratakis, K. Tian, R. A. Kishek, I. Haber, M. Reiser, and P. G. O'Shea, Phys. Plasmas 14, 120703 (2007).

[35] D. Stratakis, R. A. Kishek, R. B. Fiorito, K. Tian, I. Haber, P. G. O'Shea, M. Reiser, and J. C. T. Thangaraj, Phys. Rev. ST Accel. Beams 12, 020101 (2009). 